Mathematical Research Letters 3, 149-166 (1996)

\title{
A STABILIZATION THEOREM FOR HERMITIAN FORMS AND APPLICATIONS TO HOLOMORPHIC MAPPINGS
}

\author{
David W. Catlin and John P. D'Angelo
}

\section{Introduction}

We consider positivity conditions both for real-valued functions of several complex variables and for Hermitian forms. We prove a stabilization theorem relating these two notions, and give some applications to proper mappings between balls in different dimensions. The technique of proof relies on the simple expression for the Bergman kernel function for the unit ball and elementary facts about Hilbert spaces. Our main result generalizes to Hermitian forms a theorem proved by Polya [HLP] for homogeneous real polynomials, which was obtained in conjunction with Hilbert's seventeenth problem. See $[H]$ and $[R]$ for generalizations of Polya's theorem of a completely different kind. The flavor of our applications is also completely different.

We begin by describing our main result. Let $\mathbf{C}^{n}$ denote complex Euclidean space of $n$ dimensions, with the complex Euclidean squared norm $\|z\|^{2}=\sum_{j=1}^{n}\left|z_{j}\right|^{2}$. Suppose that $f: \mathbf{C}^{n} \rightarrow \mathbf{R}$ is a polynomial in the variables $z$ and $\bar{z}$, and that it is homogeneous of the same degree $m$ in each of these variables. We write $f(z, \bar{z})=\sum_{|\alpha|=m} \sum_{|\beta|=m} c_{\alpha \beta} z^{\alpha} \bar{z}^{\beta}$. The condition that $f$ take only real values is equi-valent to $c_{\alpha \beta}=\overline{c_{\beta \alpha}}$. We call the Hermitian matrix $\left(c_{\alpha \beta}\right)$ the underlying matrix of coefficients. Then we have the following conclusion. The function $f$ achieves a positive minimum value on the unit sphere if and only if there is an integer $d$ so that the matrix $\left(E_{\mu \nu}\right)$ is positive definite, where $\left(E_{\mu \nu}\right)$ is the underlying matrix of coefficients for the function $\|z\|^{2 d} f(z, \bar{z})$, that is

$$
\|z\|^{2 d} f(z, \bar{z})=\sum E_{\mu \nu} z^{\mu} \bar{z}^{\nu}
$$

Received November 16, 1995.

The first author is partially supported by NSF grant DMS 94015480.

The second author is partially supported by NSF grant DMS 9304580 at IAS and by MSRI. 
Consequently there is a homogeneous holomorphic vector-valued polynomial $g$ such that

$$
\|z\|^{2 d} f(z, \bar{z})=\|g(z)\|^{2} .
$$

When $\left(c_{\alpha \beta}\right)$ is itself positive definite, then $f$ must be positive on the sphere; this is the case $d=0$. In general the smallest possible value for the integer $d$ depends on the original underlying matrix of coefficients. We note that, once the form is positive for some $d$, it remains positive for all larger values, and this suggests the name "stabilization". In case the original underlying matrix of coefficients is diagonal, this theorem implies the classical theorem of Polya in the real case. Even in the diagonal case the smallest possible integer $d$ can be arbitrarily large. See Example 2 and Remark 1.

It is elementary that when the underlying matrix of coefficients is positive definite the underlying polynomial is the squared norm of a holomorphic mapping. We conclude that, although $f(z, \bar{z})$ may not be the squared norm of a holomorphic mapping, for sufficiently large integers $d$, $\|z\|^{2 d} f(z, \bar{z})$ is such a squared norm $\|g(z)\|^{2}$. Furthermore this function vanishes only at the origin.

We next indicate how this result applies to holomorphic mappings. In Theorem 3 we let $f$ be an arbitrary polynomial which is positive on the sphere; using Theorem 1 we construct a holomorphic polynomial mapping $g$ (finite-dimensionally valued) such that $f(z, \bar{z})=\|g(z)\|^{2}$ on the sphere. Theorem 3 easily implies Theorem 2 . In Theorem 2 , we consider a holomorphic polynomial mapping $p$ whose Euclidean norm on the closed ball in $\mathbf{C}^{n}$ is everywhere less than unity. Then we can find a holomorphic polynomial mapping $g$ such that $p \oplus g$ maps the sphere to some sphere; in other words, $p$ equals some of the components of a proper holomorphic polynomial mapping between balls. We must allow the target dimension to be arbitrarily large. This cannot be improved, because even in the homogeneous case, the (minimum) embedding dimension of $p \oplus g$ depends on the integer $d$ from Theorem 1. In Theorem 4, we give a result on allowable denominators for rational proper mappings between balls.

We also interpret Theorem 1 in terms of tensor products of mappings. If $h$ and $g$ are vector valued functions, we define their tensor product $h \otimes g$ to be the mapping whose components are all possible products $h_{j} g_{k}$ of the components of $h$ and $g$, in some determined order. Then $\|z\|^{2 d}=$ $\left\|H_{d}(z)\right\|^{2}$ for a certain holomorphic mapping $H_{d}$, namely the $d$-fold tensor product of the identity mapping with itself. See [D1] for many uses of this mapping. We observe that we may always write a real-valued polynomial $p$ as $p(z, \bar{z})=\|P(z)\|^{2}-\|N(z)\|^{2}$ for holomorphic mappings $P$ and $N$. 
Theorem 1 then implies that $p$ is positive on the sphere if and only if there is an integer $d$ so that we can write

$$
H_{d} \otimes N=L\left(H_{d} \otimes P\right)
$$

where $L$ is a linear transformation in the unit ball of the space of linear transformations. See equivalence 5 of Theorem 1 for the precise statement.

It is possible to phrase our result in terms of Hermitian metrics on powers of the hyperplane section bundle of complex projective space, but we do not do this here. Because of its interpretation involving tensor products, our statement might be related to the famous theorem of Kodaira on the embedding of Hodge manifolds into complex projective spaces, where one takes sufficiently high tensor powers of a positive line bundle. See [GH]. We do not pursue this here. At the close of the paper we briefly discuss the geometric meaning of the main theorem in terms of the Veronese mapping.

We close the introduction by giving another interpretation. Letting the $n$-torus act on the sphere, we see that the positivity of $f$ as a function shows that certain trigonometric polynomials are positive. By the easy direction of Bochner's theorem characterizing functions of positive type, all the matrices in a certain family must be positive definite. We relate the matrix entries of the underlying form for $\|z\|^{2 d} f(z, \bar{z})$ to the matrices in this family when $d$ is sufficiently large. This argument relies on the elementary observation that

$$
\frac{1}{(\mu-\alpha) !}=\frac{\mu^{\alpha}+p_{m-1}(\mu)}{\mu !}
$$

where $|\alpha|=m$ and $p_{m-1}$ is of degree $m-1$, and on resulting inequalities for large $|\mu|$. See Corollary 1.

The second author acknowledges both the Institute for Advanced Study and the Mathematical Sciences Research Institute for support during the academic year 1993-4. During that time he had productive conversations with many mathematicians, but he particularly thanks Andy Nicas for discussions about the mathematics in this paper. The first author acknowledges support from the NSF.

\section{Preliminaries}

\section{I.1 Notation and linear algebra}

We begin by introducing notation and recalling some elementary linear algebra. 
We often use multi-index notation in this paper, usually without much comment. If $\alpha$ is an $n$-tuple of non-negative integers, then we write $|\alpha|=$ $\sum \alpha_{j}$ as usual. We write $\alpha !=\prod \alpha_{j}$ !. As usual $z^{\alpha}=\prod z_{j}^{\alpha_{j}}$. Thus superscripts are powers, rather than being upper indices. We let $T$ denote the $n$-dimensional torus, and we let $d \theta$ denote the invariant measure normalized so that $\int_{T} d \theta=1$. We let $\theta=\left(\theta_{1}, \ldots, \theta_{n}\right)$ be an element of $T$. Thus $e^{i \alpha \theta}$ means $\prod e^{i \alpha_{j} \theta_{j}}$.

It will be important in this paper to distinguish holomorphic polynomials from arbitrary polynomials. To help do so we sometimes write $h(z)$ when $h$ is holomorphic, and $f(z, \bar{z})$ when $f$ is arbitrary. Suppose that $g$ is a vector-valued holomorphic mapping. We write $\mathbf{V}(g)=\{0\}$ to denote that the set of common zeroes of the components of $g$ consists of the origin alone. The most important holomorphic mapping used here is $H_{d}$; as mentioned in the introduction it is the tensor product of the identity mapping with itself $d$ times. In coordinates the components of $H_{d}$ are the monomials $c_{\alpha} z^{\alpha}$ where $|\alpha|=d$ and $\left|c_{\alpha}\right|^{2}=\frac{d !}{\alpha !}$ of degree $d$. Note that $\mathbf{V}\left(H_{d}\right)=\{0\}$. The components of $H_{d}$ are a basis for the complex vector space $V_{d}$ of (holomorphic) homogeneous polynomials of degree $d$ in $n$ variables, although we generally use the monomials without these constants as the standard basis.

Suppose that $V$ is a finite-dimensional complex vector space, that $\zeta \in V$, and that $Q(\zeta, \bar{\zeta})=\sum_{\mu . \nu} Q_{\mu \nu} \zeta_{\mu} \bar{\zeta}_{\nu}$ is an Hermitian form. By elementary linear algebra we can always find vectors $A_{\mu}$ and $B_{\mu}$ so that

$$
Q_{\mu \nu}=\left\langle A_{\mu}, A_{\nu}\right\rangle-\left\langle B_{\mu}, B_{\nu}\right\rangle .
$$

As a consequence of (1) we see the standard result that

Lemma 1. The Hermitian matrix $Q$ is positive definite if and only if we have $Q_{\mu \nu}=\left\langle A_{\mu}, A_{\nu}\right\rangle$ where the set of vectors $\left\{A_{\mu}\right\}$ form a basis.

Lemma 1 implies the simple but fundamental statement given by Lemma 2 .

Lemma 2. Let $f=\sum \sum c_{\alpha \beta} z^{\alpha} \bar{z}^{\beta}$ be a real-valued polynomial that is homogeneous of degree $m$ in both $z$ and $\bar{z}$. Suppose that the underlying Hermitian matrix of coefficients $c_{\alpha \beta}$ has $k^{+}$positive and $k^{-}$negative eigenvalues. Then there are holomorphic vector-valued polynomials $P$ and $N$, each homogeneous of degree $m$, such that

$$
f(z, \bar{z})=\|P(z)\|^{2}-\|N(z)\|^{2} .
$$

We can always choose $P$ and $N$ to have $k^{+}$and $k^{-}$components respectively. The underlying matrix of coefficients is positive definite if and only if we may write

$$
f(z, \bar{z})=\|P(z)\|^{2}
$$


where $P=T H_{m+d}$ for some invertible linear transformation $T$. When the underlying matrix of coefficients is positive definite we also must have $\mathbf{V}(P)=\{0\}$.

Proof. By elementary linear algebra as in (1) we may find vectors so that $c_{\alpha \beta}=\left\langle A_{\alpha}, A_{\beta}\right\rangle-\left\langle B_{\alpha}, B_{\beta}\right\rangle$. Then we define $P$ by $P(z)=\sum A_{\alpha} z^{\alpha}$ and $N$ by $N(z)=\sum B_{\alpha} z^{\alpha}$. Easy computation then yields (2) and the result about the number of eigenvalues. The eigenvalues are all positive if and only if $\left(c_{\alpha \beta}\right)$ is positive definite. Then the vectors $A_{\alpha}$ can be chosen to be linearly independent. Thus the underlying matrix is positive definite if and only if the matrix $\left(A_{\beta \alpha}\right)$ is invertible, where we write $A_{\alpha}=\sum A_{\beta \alpha} e_{\beta}$ in terms of the standard orthonormal basis. This implies the statement about $T$ as well, because $H_{m+d}$ includes all monomials of degree $m+d$. It remains to prove that $\mathbf{V}(P)=\{0\}$. Suppose that there exists $w$ with $P(w)=0$. Then

$$
0=\|P(w)\|^{2}=\sum\left\langle A_{\alpha}, A_{\beta}\right\rangle w^{\alpha} \bar{w}^{\beta}=0 .
$$

The underlying Hermitian form then has a non-trivial kernel unless $w^{\alpha}$ must vanish for all $\alpha$. Hence, if it is positive definite, the variety must consist of the origin alone.

Example 1. When $f(z, \bar{z})=\|P(z)\|^{2}$, and $\mathbf{V}(P)=\{0\}$, the underlying form might be only semi-definite. Consider the simple example $\left|z_{1}\right|^{4}+$ $\left|z_{2}\right|^{4}$. The underlying form is diagonal, with eigenvalues $1,0,1$. Theorem 1 implies however that we can obtain a positive definite underlying form by multiplying by the squared norm.

\section{I.2 Positivity conditions}

Next we study positivity (away from the origin) of $f$ as a function. Suppose that $f$ is our given bihomogeneous polynomial, and that $f(z, \bar{z})>$ 0 for $z \neq 0$. Simple examples (see example III.2) reveal that the underlying matrix of coefficients can have eigenvalues of both signs, so the Hermitian form on $V_{m}$ defined by this matrix need not be positive. Nevertheless the positivity of $f$ as a function has many consequences.

By compactness there is an $\epsilon>0$ such that $f(z, \bar{z}) \geq \epsilon$ on the unit sphere. By homogeneity we have

$$
f(z, \bar{z}) \geq \epsilon\|z\|^{2 m}
$$

everywhere. Now let the torus $T$ act on the sphere. This implies that, for all $\theta$,

$$
g(\theta)=\sum c_{\alpha \beta} z^{\alpha} \bar{z}^{\beta} e^{i \theta(\alpha-\beta)} \geq \epsilon\|z\|^{2 m}
$$


as well. Integrating over the torus reveals that $\sum c_{\alpha \alpha}|z|^{2 \alpha} \geq \epsilon|| z||^{2 m}$ holds. To obtain more general inequalities we define $A_{\mu \nu}$ by

$$
A_{\mu \nu}(z, \bar{z})=A_{\mu \nu}=\sum_{\beta} c_{(\beta+\mu-\nu) \beta} z^{\beta+\mu-\nu} \bar{z}^{\beta}
$$

Note that $A_{\mu \nu}(z, \bar{z})=\int_{T} e^{-i(\mu-\nu) \theta} g(\theta) d \theta$.

Proposition 1. Suppose that (3) holds. For all non-zero $z$ the matrix $A_{\mu \nu}$ is positive definite and its minimum eigenvalue is at least $\epsilon\|z\|^{2 m}$.

Proof. We estimate $\sum A_{\mu \nu} \zeta_{\mu} \bar{\zeta}_{\nu}$ as follows.

$$
\begin{aligned}
\sum A_{\mu \nu} \zeta_{\mu} \bar{\zeta}_{\nu} & =\sum \int_{T} \zeta_{\mu} \bar{\zeta}_{\nu} e^{-i(\nu-\mu) \theta} g(\theta) d \theta \\
& =\int_{T}\left|\sum_{\mu} e^{-i \mu \theta} \zeta_{\mu}\right|^{2} g(\theta) d \theta \\
& \geq \int_{T} \epsilon\|z\|^{2 m}\left|\sum_{\mu} e^{-i \mu \theta} \zeta_{\mu}\right|^{2} d \theta \\
& =\epsilon\|z\|^{2 m} \int_{T}\left|\sum_{\mu} e^{-i \mu \theta} \zeta_{\mu}\right|^{2} d \theta \\
& =\epsilon\|z\|^{2 m}|| \zeta \|^{2}
\end{aligned}
$$

The inequality comparing the first and last expression proves the proposition.

Next we make a simple observation that will enable us to relate these matrices to values of $f$.

Observation. Let $\mu$ and $\alpha$ be multi-indices, and suppose that $|\alpha|=m$. Then there is a polynomial $p_{m-1}$ on $\mathbf{R}^{n}$ of degree $m-1$ such that $\frac{1}{(\mu-\alpha) !}-$ $\frac{\mu^{\alpha}}{\mu !}=\frac{p_{m-1}(\mu)}{\mu !}$. Hence there is a constant $C>0$ so that $\left|\frac{1}{(\mu-\alpha) !}-\frac{\mu^{\alpha}}{\mu !}\right|<$ $C \frac{|\mu|^{m-1}}{\mu !}$.

Proposition 2. Given $\epsilon>0$, there is an $N_{0}$ so that $|\mu|>N_{0}$ implies that

$$
\left|\frac{1}{(\mu-\alpha) !}-\frac{\mu^{\alpha}}{\mu !}\right|<\epsilon|\mu|^{m}
$$

Proof. This follows immediately from the observation and the definition of the limit, applied to $\lim _{|\mu| \rightarrow \infty} \frac{p_{m-1}(\mu)}{|\mu|^{m}}=0$. 


\section{I.3 Elementary Hilbert space considerations}

We now recall some facts about the Hilbert space of holomorphic square integrable functions on the unit ball $B_{n}$. First of all, the Bergman kernel function $K(z, \bar{\zeta})$ for a bounded domain $\Omega$ is the kernel of the integral operator that projects the square integrable functions $L^{2}(\Omega)$ onto the closed subspace $A^{2}(\Omega)$ of holomorphic square integrable functions. It satisfies $K(z, \bar{\zeta})=\sum \phi_{\alpha}(z) \bar{\phi}_{\alpha}(\zeta)$ where $\left\{\phi_{\alpha}\right\}$ is a complete orthonormal set for $A^{2}(\Omega)$. For the unit ball (and a few other domains) normalized monomials form such a complete orthonormal set and the series can be summed explicitly. For the unit ball $B_{n}$ the result is that $K(z, \bar{\zeta})=\frac{n !}{\pi^{n}}(1-\langle z, \zeta\rangle)^{-(n+1)}$. We can decompose $A^{2}=A^{2}\left(B_{n}\right)$ as an orthogonal sum $A^{2}\left(B_{n}\right)=\sum V_{k}$ where $V_{k}$ is the subspace of holomorphic homogeneous polynomials of degree $k$.

We say that a linear operator $T: L^{2} \rightarrow L^{2}$ is positive if there is a positive number $c$ so that $\langle T g, g\rangle \geq c\langle g, g\rangle$. Here the inner product is the usual one given by integration.

Lemma 3. Suppose that $\left(c_{\alpha \beta}\right)$ is the matrix of an Hermitian form on $V_{m}$. Let $f(z, \bar{\zeta})=\sum c_{\alpha \beta} z^{\alpha} \bar{\zeta}^{\beta}$ be the corresponding bihomogeneous polynomial. Let $R: A^{2} \rightarrow A^{2}$ be the operator defined by

$$
R g(z)=\int_{B_{n}} f(z, \bar{\zeta}) g(\zeta) d V(\zeta)
$$

Then $R$ is the zero operator on $V_{m}^{\perp}$ and $R$ maps $V_{m}$ to itself. Furthermore $\left(c_{\alpha \beta}\right)$ is positive definite if and only if the operator $R$ is positive on $V_{m}$.

Proof. We expand $g$ as a convergent power series, writing $g(\zeta)=\sum g_{\gamma} \zeta^{\gamma}$. We compute

$$
R g(z)=\int \sum_{\alpha, \beta} \sum_{\delta} c_{\alpha \beta} z^{\alpha} \bar{\zeta}^{\beta} g_{\delta} \zeta^{\delta} d V(\zeta)
$$

By the orthogonality of the monomials in $A^{2}\left(B_{n}\right)$ we get zero unless $\beta=\delta$. This yields

$$
R g(z)=\sum_{\alpha, \beta} c_{\alpha \beta} z^{\alpha} g_{\beta}\left\|z^{\beta}\right\|_{A^{2}}^{2}
$$

and hence proves the first statement. Writing $d_{\alpha}=\left\|z^{\alpha}\right\|_{A^{2}}^{2}$, we see that

$$
\langle R g, g\rangle=\iint \sum_{\alpha, \beta} \sum_{\gamma, \delta} c_{\alpha \beta} z^{\alpha} \bar{\zeta}^{\beta} g_{\delta} \zeta^{\delta} \bar{g}_{\gamma} \bar{z}^{\gamma} d V(z) d V(\zeta)=\sum_{\alpha, \beta} c_{\alpha \beta} d_{\alpha} d_{\beta} \bar{g}_{\alpha} g_{\beta}
$$


Thus, as a form on $V_{m}, R$ has underlying matrix $\left(c_{\beta \alpha} d_{\alpha} d_{\beta}\right)$. This is positive if and only if $\left(c_{\alpha \beta}\right)$ is, because of Lemma 1 , and because the $d_{\alpha}$ are positive numbers.

We continue to let $f=\sum c_{\alpha \beta} z^{\alpha} \bar{\zeta}^{\beta}$ be our given bihomogeneous polynomial. Let $R_{k+m}$ denote the operator on $A^{2}\left(B_{n}\right)$ whose kernel is $\langle z, \zeta\rangle^{k} f(z, \bar{\zeta})$. The main point of Theorem 1 is to show that $f$ positive as a function implies for sufficiently large $d$ that $\|z\|^{2 d} f(z, \bar{z})$ has a positive definite underlying form. By Lemma 3, it suffices to show that $R_{d+m}$ is a positive operator on $V_{m+d}$ for sufficiently large $d$.

\section{Statement and proof of Theorem 1}

We are now prepared to give equivalent conditions for the positivity of a homogeneous polynomial on the sphere. Theorem 1 is the principal result of this paper.

Theorem 1. Let $f(z, \bar{z})=\sum \sum c_{\alpha \beta} z^{\alpha} \bar{z}^{\beta}$ be a real-valued polynomial that is homogeneous of degree $m$ in $z$ and also in $\bar{z}$. The following are equivalent.

1) $f$ achieves a positive minimum value on the sphere.

2) There is an integer d such that the underlying Hermitian matrix for $\|z\|^{2 d} f(z, \bar{z})$ is positive definite. Thus

$$
\|z\|^{2 d} f(z, \bar{z})=\sum E_{\mu \nu} z^{\mu} \bar{z}^{\nu}
$$

where $\left(E_{\mu \nu}\right)$ is positive definite.

3) There is an integer $d$ such that the operator $R_{m+d}$ defined by the kernel $k_{d}(z, \zeta)=\langle z, \zeta\rangle^{d} f(z, \bar{\zeta})$ is a positive operator from $V_{m+d} \subset A^{2}\left(B_{n}\right)$ to itself.

4) There is an integer $d$ and a holomorphic homogeneous vector-valued polynomial $g$ of degree $m+d$ such that $\mathbf{V}(g)=\{0\}$ and such that $\|z\|^{2 d} f(z, \bar{z})=\|g(z)\|^{2}$.

5) Write $f(z, \bar{z})=\|P(z)\|^{2}-\|N(z)\|^{2}$ for holomorphic homogeneous vector-valued polynomials $P$ and $N$ of degree $m$. Then there are an integer $d$ and a linear transformation $L$ such that the following are true:

5.1) $I-L^{*} L$ is positive semi-definite.

5.2) $H_{d} \otimes N=L\left(H_{d} \otimes P\right)$.

5.3) $\mathbf{V}\left(\sqrt{I-L^{*} L}\left(H_{d} \otimes P\right)\right)=\{0\}$.

Proof. The hard implication is that 1) implies 3 ), so we do this last. It follows immediately from Lemma 3 that 2) and 3) are equivalent. We next observe that 2) implies 4), immediately from Lemma 1 . To prove that 2) implies 5), we recall that $\|g \otimes h\|^{2}=\|g\|^{2}\|h\|^{2}$ and also that $\|z\|^{2 d}=$ 
$\left\|H_{d}(z)\right\|^{2}$. We plug these into $\|g(z)\|^{2}=\|z\|^{2 d}\left(\|P(z)\|^{2}-\|N(z)\|^{2}\right)$, which is part of the hypothesis. After moving terms we obtain

$$
\left\|H_{d} \otimes N\right\|^{2}+\|g\|^{2}=\left\|H_{d} \otimes P\right\|^{2} .
$$

Thus $\left(H_{d} \otimes N\right) \oplus g$ and $H_{d} \otimes P$ are holomorphic mappings with the same squared norms. Hence, see [D1], after perhaps including enough zero components, there is a constant unitary matrix $U$ such that $\left(H_{d} \otimes N\right) \oplus g=$ $U\left(H_{d} \otimes P\right)$. Writing this matrix in terms of blocks, one of which is $L$, we obtain submatrices $L$ and $\sqrt{I-L^{*} L}$ such that 5.1), 5.2), and 5.3) hold. Notice that we obtain the formula that $g=\sqrt{I-L^{*} L}\left(H_{d} \otimes P\right)$. Thus 4) and 5) are equivalent, without passing through 1).

That 5) implies 1) is also easy. Given 5), define $g$ by $g=\sqrt{I-L^{*} L}\left(H_{d} \otimes\right.$ $P)$. Then 5.2) and the definition of $f$ imply that

$$
\|z\|^{2 d}\|N(z)\|^{2}+\|g(z)\|^{2}=\|z\|^{2 d}\|P(z)\|^{2} .
$$

We see immediately that, on the sphere, $\|N(z)\|^{2}+\|g(z)\|^{2}=\|P(z)\|^{2}$. Recalling that $f(z, \bar{z})=\|P(z)\|^{2}-\|N(z)\|^{2}$, we see that the minimum value of $f$ on the sphere equals the minimum value of $g$ on the sphere, which is positive because $\mathbf{V}(g)=\{0\}$ by 5.3).

Thus the theorem follows if we can show that 1) implies 3). Let $S$ be the operator on $A^{2}$ whose kernel is $K(z, \zeta) f(z, \zeta)$. We claim that there are positive constants $c_{j, n}$ such that $c_{j, n} R_{j}$ equals the restriction of $S$ to $V_{j}$. The orthogonality of the spaces $V_{j}$ shows that $R_{j}$ is the zero operator off $V_{j}$. Therefore the power series expansion

$$
(1-\langle z, \zeta\rangle)^{-n-1}=\sum\left(\begin{array}{c}
j+n \\
j
\end{array}\right)\langle z, \zeta\rangle^{j}
$$

implies the claim. To prove that $R_{d+m}$ is a positive operator on $V_{m+d}$ for sufficiently large $d$, it therefore suffices to prove that the operator $S$ has only finitely many non-positive eigenvalues.

It remains only to show that $\langle S g, g\rangle \geq c\|g\|_{A^{2}}^{2}$ for $g \in V_{j}$ for sufficiently large $j$. We introduce the operator $T$ on $L^{2}$, whose kernel is $K(z, \zeta) f(z, \bar{z})$. Then we have

$$
\langle T g, g\rangle=\iint K(z, \zeta) f(z, \bar{z}) \bar{g}(z) g(\zeta) d V(\zeta) d V(z)=\int f(z, \bar{z})|g(z)|^{2} d V(z)
$$

by the reproducing property of the Bergman kernel. If we now introduce a cut-off function $\phi$ that is positive at the origin, and invoke the positivity of 
$f$ as a function, we obtain that $\langle(T+\phi) g, g\rangle \geq c\|g\|_{L^{2}}^{2}$. Here $\phi$ is smooth, non-negative, positive at the origin, and has compact support in the ball.

Next we write the operator equation $S=(T+\phi)+(E-\phi)$ where $E=S-T$. We observe that the kernel of $E$ vanishes at $z=\zeta$. Since this compensates for the singularity of $K(z, \zeta)$ at $z=\zeta$ on the boundary of the ball, $E$ is a compact operator. Since $\phi$ is smooth and compactly supported we also have that $E-\phi$ is a compact operator. Since $E-\phi$ is a compact operator on $L^{2}$, we can approximate it in norm by an operator $L$ with finite-dimensional range. See [Ru] (pages 97-107) for the necessary statements about compact operators. We write $\||| L|| \mid$ for the operator norm of $L$.

Then $S=(T+\phi)+(E-\phi-L)+L$. Write $L_{j}$ for the restriction of $L$ to $V_{j}$. Because $L$ has finite-dimensional range, $\left\|\left|L_{j}\right|\right\|<c / 3$ for $j$ sufficiently large. Also we may assume that $\left\||E-\phi-L \||<c / 3\right.$. Therefore, on $V_{j}$ for sufficiently large $j$, the operator $S$ is a small perturbation of $T+\phi$. Therefore $S$ is also a positive operator on $V_{j}$ for sufficiently large $j$. Hence $S$ has only finitely many non-positive eigenvalues, so $R_{d+m}$ is a positive operator on $V_{m+d}$ for $d$ sufficiently large. This proves that 1) implies 3 ) and completes the proof.

The proof of Theorem 1 doesn't give a value for $d$; the value of $d$ is useful because it is closely related to the embedding dimensions of mappings discussed in the next section, and because there is interest in the analogous exponent in the classical theorem of Polya $[\mathrm{R}]$. We therefore relate the numbers $E_{\mu \nu}$ to the numbers $c_{\alpha \beta}$ and to the matrices $A_{\mu \nu}$ from Proposition 1 .

Corollary 1. Let $f(z, \bar{z})=\sum c_{\alpha \beta} z^{\alpha} \bar{z}^{\beta}$ be a bihomogeneous polynomial that is positive away from the origin. There is an integer $N$ such that the matrix $L_{\mu \nu}$ defined for $|\mu|=|\nu|=N$ by

$$
L_{\mu \nu}=\sum_{\alpha-\beta=\mu-\nu} c_{\alpha \beta}\left(\frac{\mu}{|\mu|}\right)^{\alpha}\left(\frac{\nu}{|\nu|}\right)^{\beta}
$$

is positive definite. Similarly there is an integer $N^{\prime}$ so that the matrix

$$
A_{\mu \nu}\left(\sqrt{\frac{\nu}{|\nu|}}, \sqrt{\frac{\nu}{|\nu|}}\right)=\sum_{\alpha-\beta=\mu-\nu} c_{\alpha \beta}\left(\frac{\nu}{|\nu|}\right)^{\alpha+\beta}
$$

is positive definite when $|\mu|=|\nu|=N^{\prime}$.

Proof. For each positive integer $d$ we write $E_{\mu \nu}(d)$ for the underlying matrix of coefficients for the function

$$
\|z\|^{2 d} f(z, \bar{z}) .
$$


Then the multinomial expansion and interchange of summation yields

$$
E_{\mu \nu}(d)=d ! \sum_{\alpha-\beta=\mu-\nu} c_{\alpha \beta} \frac{1}{(\mu-\alpha) !(\nu-\beta) !} .
$$

We put $|\mu|=|\nu|=N$ and rewrite using the observation to get

$$
\begin{aligned}
& E_{\mu \nu}(d)= \\
& \quad \frac{N !}{N^{m}+p_{m-1}(N)} \sum_{\alpha-\beta=\mu-\nu} c_{\alpha \beta}\left(\mu^{\alpha}+p_{m-1}(\mu)\right)\left(\nu^{\beta}+p_{m-1}(\nu)\right) \frac{1}{\sqrt{\mu ! \nu !}} .
\end{aligned}
$$

Next we multiply and divide by $N^{m}$ to get

$$
E_{\mu \nu}(d)=
$$

$$
\frac{N !}{\sqrt{\mu ! \nu !}(1+O(1 / N))} \sum_{\alpha-\beta=\mu-\nu} c_{\alpha \beta}\left(\frac{\mu}{|\mu|}+O(1 / N)\right)^{\alpha}\left(\frac{\nu}{|\nu|}+O(1 / N)\right)^{\beta} .
$$

Theorem 1 implies that this matrix is positive definite when $d$ is sufficiently large. By Lemma 1 we do not alter the positive definiteness if we divide by $\frac{N !}{\sqrt{\mu ! \nu !}}$, obtaining

$$
D_{\mu \nu}(d)=\frac{1}{(1+O(1 / N))} \sum_{\alpha-\beta=\mu-\nu} c_{\alpha \beta}\left(\frac{\mu}{|\mu|}+O(1 / N)\right)^{\alpha}\left(\frac{\nu}{|\nu|}+O(1 / N)\right)^{\beta} .
$$

Now it is clear that, for sufficiently large $|N|$, the matrix (5) is positive definite if and only if

$$
\sum_{\alpha-\beta=\mu-\nu} c_{\alpha \beta}\left(\frac{\mu}{|\mu|}\right)^{\alpha}\left(\frac{\nu}{|\nu|}\right)^{\beta}
$$

is positive definite. This proves the first statement.

To prove the second, replace $\mu$ by $\mu=\nu+\alpha-\beta$ in the term $\left(\frac{\mu}{|\mu|}+\right.$ $O(1 /|N|))^{\alpha}$ arising in (5). Since the multi-indices $\alpha$ and $\beta$ have fixed length $m$, we see that $\frac{\mu+\alpha-\beta}{|\mu|}$ can be also written as $\frac{\nu}{|\nu|}+O(1 / N)$. Thus the matrix $A_{\mu \nu}\left(\sqrt{\frac{\nu}{|\nu|}}, \sqrt{\frac{\nu}{|\nu|}}\right)$ is also positive definite for sufficiently large $N$. This proves the Corollary.

Observe that Proposition 1 guarantees that $A_{\mu \nu}(z, \bar{z})$ is positive definite for each $z$. In Corollary 1 however the entries are evaluated at points depending on the indices. Corollary 1 enables one to obtain quantitative information on the integer $d$ without explicitly performing the multiplication by $\|z\|^{2 d}$, but we do not develop this reasoning further here. 


\section{Examples and applications}

We first observe that the smallest possible value of the integer $d$ from Theorem 1 can be arbitrarily large.

Example 2. Consider the one parameter family of polynomials $p_{c}$ on $\mathbf{C}^{2}$ with variables $(z, w)$ defined by

$$
p_{c}(z, w, \bar{z}, \bar{w})=|z|^{4}+|w|^{4}-c|z w|^{2} .
$$

Here $c$ is a real number. Then $p_{c}$ is positive on the sphere if and only if $c<2$. The original underlying matrix is diagonal, with eigenvalues of $1,-c, 1$. After multiplying through by $\left(|z|^{2}+|w|^{2}\right)^{d}$ we also obtain diagonal matrices $L_{d}$ of size $d+3$ by $d+3$. If we let $d(c)$ denote the minimum $d$ for which $L_{d}$ is positive definite, then we compute that $d(c)>(3 c-2) /(2-c)$ and hence that $\lim _{c \rightarrow 2} d(c)=\infty$.

This example indicates that the computations from Theorem 1 are easier when the original underlying matrix is diagonal. In fact this special case is equivalent to the theorem of Polya [HLP]. Polya began with a homogeneous polynomial in several real variables, assumed to be positive on the positive part of the hyperplane defined by $|x|=\sum x_{j}=1$. He proved that there is an integer $d$ so that

$$
|x|^{d} p(x)=q(x)
$$

where all the coefficients of $q$ are positive. The value of this theorem is that $p$ is then expressed as a quotient of polynomials with positive coefficients. When the underlying matrix of coefficients is diagonal, Theorem 1 implies Polya's theorem, and conversely. The correspondence between them is given by writing $x_{j}=\left|z_{j}\right|^{2}$ and observing that a diagonal matrix is positive definite if and only if all the diagonal elements are positive. Our proof of Theorem 1 yields a new proof of Polya's theorem. On the other hand the computations in Corollary 1 yield a direct proof, which we include now.

\section{Proof of Polya's theorem}

Put $f(x)=\sum c_{\alpha} x^{\alpha}$. Then

$$
|x|^{d} f(x)=d ! \sum_{\mu}\left(\sum_{\alpha} \frac{c_{\alpha}}{(\mu-\alpha) !}\right) x^{\mu} .
$$

By the observation we can write the coefficient of $x^{\mu}$ as

$$
\frac{d !}{\mu !}\left(f(\mu)+p_{m-1}(\mu)\right)
$$


Suppose that $f(\mu) \geq \epsilon|\mu|^{m}$. By Proposition 2 we can choose $|\mu|$ sufficiently large that $\left|p_{m-1}(\mu)\right|<\frac{\epsilon}{2}|\mu|^{m}$. Then $f(\mu)+p_{m-1}(\mu)>\frac{\epsilon}{2}|\mu|^{m}$ and the result follows.

Remark 1. The theorem fails in the positive semi-definite case. In other words, the following is true. There exist real-valued polynomials $f(z, \bar{z})$ that are non-negative as functions, and for which the underlying Hermitian form for $\|z\|^{2 d} f(z, \bar{z})$ has a negative eigenvalue for every $d$. One example is $|z|^{4}-2|z w|^{2}+|w|^{4}=\left(|z|^{2}-|w|^{2}\right)^{2}$.

Next we give a concrete corollary obtained by applying the theorem when $n=2$. Suppose that $C=\left(c_{a b}\right)$ is a Hermitian symmetric matrix of size $m+1$. We define a new matrix $E(C)$ by the following operation: $E(C)=C \oplus 0+0 \oplus C$. This means that we augment $C$ by including a leading row and column of zeroes, augment $C$ by including a last row and column of zeroes, and add the two matrices to get a Hermitian symmetric matrix of size $m+2$. Then Theorem 1 gives a necesssary and sufficient condition for there to be an integer $d$ for which $E^{d}(C)$ is positive definite.

Corollary 2. There is an integer $d$ for which $E^{d}(C)$ is positive definite if and only if $\sum_{a=0}^{m} \sum_{b=0}^{m} c_{a b} t^{a} \bar{t}^{b}>0$ for all non-zero complex numbers $t$.

Proof. Replace $t$ by $z / w$ in the given condition, and multiply through by $|w|^{2 m}$. The condition then becomes

$$
\sum_{a=0}^{m} \sum_{b=0}^{m} c_{a b} z^{a} w^{m-a} \bar{z}^{b} \bar{w}^{m-b}>0 .
$$

Thus we have a bihomogeneous polynomial in two variables. On the other hand, the operation $E$ is easily seen to be equivalent to multiplying the function defining the underlying matrix of coefficients by $|z|^{2}+|w|^{2}$, and recomputing the underlying matrix of coefficients of the product. Thus the result follows from Theorem 1.

It may be worth remarking that the special case of Corollary 2 when the matrix is diagonal is a version of the Polya theorem for inhomogeneous polynomials in one variable.

\section{Applications to proper mappings between balls}

We give several applications to proper mappings between balls. We consider two questions. First, suppose that $p: \mathbf{C}^{n} \rightarrow \mathbf{C}^{k}$ is a (holomorphic) polynomial mapping and that $\|p(z)\|<1$ for all $z$ in the closed unit ball. We will show that $p$ consists of some of the components of a proper mapping 
from $B_{n}$ to some $B_{N}$. We prove in Theorem 2 that there is a positive integer $N-k$ and a polynomial mapping $g: \mathbf{C}^{n} \rightarrow \mathbf{C}^{N-k}$ such that $p \oplus g$ is the required mapping. In Theorem 4 we suppose that $q$ is a given polynomial function with $q(0)=0$ and that $|q(z)|<1$ on the closed ball. We prove that there is a rational proper mapping, reduced to lowest terms, with denominator $1+q$.

Theorem 2. Suppose that $p$ is a homogeneous vector-valued polynomial on $\mathbf{C}^{n}$ and that $\|p(z)\|^{2}<1$ on the unit sphere. Then there is a polynomial mapping $g$ such that $p \oplus g$ defines a proper holomorphic mapping between balls.

We derive Theorem 2 from the following related result.

Theorem 3. . Suppose that $f(z, \bar{z})$ is a real-valued polynomial of degree $m$ (not necessarily homogeneous), and that $f(z, \bar{z})>0$ on the unit sphere. Then there is a (holomorphic) polynomial mapping $g$ with finitedimensional range such that $f(z, \bar{z})=\|g(z)\|^{2}$ on the unit sphere.

Proof of Theorem 2 assuming Theorem 3. The result is obvious if $p$ is a constant. Given a non-constant $p$, we consider $f(z, \bar{z})=\|z\|^{2 m}-p(z, \bar{z})$. Then $f$ satisfies the hypotheses of Theorem 3 , so that $f(z, \bar{z})=\|g(z)\|^{2}$ on the sphere for some $g$. Then $p \oplus g$ is a non-constant holomorphic polynomial mapping whose squared norm $\|p\|^{2}+\|g\|^{2}$ equals unity on the sphere. By the maximum principle $p \oplus g$ is the required mapping.

Proof of Theorem 3. We are given that $f$ is degree $m$ in both $z$ and $\bar{z}$, so of total degree $2 m$. We may assume that $m$ is even, because we may replace $f$ by $\|z\|^{2} f$ otherwise without changing the hypothesis on $f$. Given $f=$ $\sum_{|\alpha|} \sum_{|\beta|} c_{\alpha \beta} z^{\alpha} \bar{z}^{\beta}$, we homogenize it by adding a new complex variable $t$, and put

$$
F(z, t, \bar{z}, \bar{t})=\sum_{\alpha} \sum_{\beta} c_{\alpha \beta} z^{\alpha} t^{m-|\alpha|} \bar{z}^{\beta} \bar{t}^{m-|\beta|} .
$$

Since $F\left(z, e^{i \theta}, \bar{z}, e^{-i \theta}\right)=f\left(z e^{-i \theta}, \bar{z} e^{i \theta}\right)$, we see that $F$ is positive when both $\|z\|^{2}=1$ and $|t|^{2}=1$. By homogeneity we then have

$$
F(z, t, \bar{z}, \bar{t}) \geq c\left(\|z\|^{2}+|t|^{2}\right)^{m}
$$

on the set $\|z\|^{2}=|t|^{2}$. We can therefore choose a constant $C$ so that, away from the origin $(0,0)$,

$$
F(z, t, \bar{z}, \bar{t})+C\left(\left(|| z||^{2}-|t|^{2}\right)^{m}>0 .\right.
$$

By Theorem 1, applied when the domain dimension is one larger, there is an integer $d$ and a holomorphic polynomial mapping $g$ such that 


$$
\left(\|z\|^{2}+|t|^{2}\right)^{d} F(z, t, \bar{z}, \bar{t})+C\left(\left(\left\|\left.z\left|\|^{2}-\right| t\right|^{2}\right)^{m}=\|g(z, t)\|^{2} .\right.\right.
$$

Setting $t=1$ to dehomogenize (8), we see that

$$
\left(\|z\|^{2}+1\right)^{d} f(z, \bar{z})+C\left(\left(\|z\|^{2}-1\right)^{m}=\|g(z, 1)\|^{2} .\right.
$$

It follows immediately from $(9)$ that $2^{d} f(z, \bar{z})=\|g(z, 1)\|^{2}$, so Theorem 3 follows.

Remark. Theorem 2 is easier when $p$ is homogeneous. One then applies Theorem 1 directly to the form by $f(z, \bar{z})=\|z\|^{2 m}-\|p(z)\|^{2}$. By statement 2 ) there is an integer $d$ and a holomorphic mapping $g$ such that

$$
\|g\|^{2}=\|z\|^{2 d}\left(\|z\|^{2 m}-\|p(z)\|^{2}\right)
$$

The holomorphic mapping $g \oplus p$ then satisfies

$\|p \oplus g\|^{2}=\|p\|^{2}+\|g\|^{2}=\|p\|^{2}+\|z\|^{2 d}|| p\left\|^{2}=\right\| z\left\|^{2(m+d)}=\right\| H_{m+d} \|^{2}=1$

on the unit sphere.

Remark. Lempert [L1, L2] has proved an analog of Theorem 3 for functions extending holomorphically past the boundary of any strongly pseudoconvex domain whose boundary is real-analytic. Løw $[\mathrm{L}]$ proved an analog of Theorem 3 for functions holomorphic on a smoothly bounded strongly pseudoconvex domain that extend continuously up to the boundary. For the particular case of the ball his result gives a version of Theorem 2 . The mapping $g$ he obtains will be finite-dimensionally valued, but not a polynomial. We repeat for emphasis that we obtain in Theorems 2 and 3 holomorphic polynomial mappings $g$ with finite-dimensional ranges.

We consider briefly the consequences that $p \oplus g$ is a proper mapping between balls when $p$ is a vector-valued polynomial of degree $m$. Then $\|p(z)\|^{2}+\|g(z)\|^{2}=1$ on the sphere. We write $p=\sum_{k=0}^{m} p_{k}$ for the decomposition of $p$ into its homogeneous parts and similarly for $g$. The tensor product operation enables us to homogenize. We have the equation (on the sphere)

$$
\sum_{k=0}^{m+d}\left\langle g_{k}, g_{l}\right\rangle+\left\langle p_{k}, p_{l}\right\rangle=\|z\|^{2(m+d)} .
$$

Replace $z$ by $e^{i \theta} z$, where now $\theta$ is on the circle. We obtain the following equations on the sphere:

$$
\sum\left(\left\|g_{k}\right\|^{2}+\left\|p_{k}\right\|^{2}\right)=\|z\|^{2(m+d)}
$$


when we consider the constant term, and

$$
\sum\left(\left\langle g_{l+s}, g_{l}\right\rangle+\left\langle p_{l+s}, p_{l}\right\rangle\right)=0
$$

for $s \neq 0$, when we equate Fourier coefficients. As in [D2] we can homogenize these equations by tensoring each $g_{k}$ or $p_{k}$ with $H_{m+d-k}$. We obtain

$$
\sum_{k}\left(\left\|H_{m+d-k} \otimes g_{k}\right\|^{2}+\left\|H_{m+d-k} \otimes p_{k}\right\|^{2}\right)=\|z\|^{2(m+d)}=\left\|H_{m+d}\right\|^{2}
$$

from the constant term, and for $s \neq 0$, the equations (10),

$$
\sum_{l}\left(\left\langle H_{m+d-l} \otimes g_{l+s}, H_{m+d-l} \otimes g_{l}\right\rangle+\left\langle H_{m+d-l} \otimes q_{l+s}, H_{m+d-l} \otimes q_{l}\right\rangle\right)=0 .
$$

Hence the conclusion of Theorem 2 implies additionally that $g$ satisfies all these orthogonality relations. In Proposition 1 from [D2], we are given an orthogonal direct sum of monomials of degree at most $m-1$. We can find a monomial of degree $m$ that makes the entire expression into a proper mapping between balls, provided a certain form is positive semi-definite. Theorem 2 reveals that the orthogonality conditions are not needed.

We next prove the theorem about allowable denominators.

Theorem 4. Suppose that $q$ is a holomorphic polynomial that satisfies $q(0)=0$ and $|q|<1$ on the unit ball in $\mathbf{C}^{n}$. Then there is some $N$ and a polynomial mapping $p: \mathbf{C}^{n} \rightarrow \mathbf{C}^{N}$ such that $\frac{p}{1+q}$ is a proper rational mapping between balls that is reduced to lowest terms.

Proof. Since $|q|<1$ on the sphere, for sufficiently small positive $\epsilon, \mid(1+$ $\epsilon) q \mid<1$ there as well. By Theorem $2(1+\epsilon) q$ is a component of a proper polynomial mapping $q \oplus g$ between balls. We consider an automorphism of the target ball (See [D1] for example) of the form $\phi_{a}(w)=\frac{w-L_{a}(w)}{1-\langle w, a\rangle}$, where $a=\frac{-1}{1+\epsilon} \oplus 0$. Then the composition $\phi_{a}(q \oplus g)$ has denominator $1+q$. By considering the numerator it is easy to prove that the map constructed in this manner is reduced to lowest terms, in the sense that $1+q$ does not divide all the components of the numerator.

Observe that the dimension $N$ depends on how close $q$ gets to unity. Wono Setya-Budhi $[\mathrm{W}]$ proved Theorem 4 in the special case when $q$ is a monomial.

In this paper we have considered forms defined by polynomials. This enables us also to consider rational mappings. The reader may wonder 
whether this may be too restrictive. Our results about proper mappings apply rather generally, because of a theorem of Forstneric. [F]. Suppose that the domain dimension is at least two, and that $f$ is a proper holomorphic mapping between balls. If $f$ is smooth (of class $C^{\infty}$ ) on the closed ball, then $f$ must be a rational mapping. For rational mappings $\frac{p}{q}$ we consider the Hermitian form defined by $\|\left. p\right|^{2}-|q|^{2}$ and the methods of this paper apply. We hope to find further applications of these ideas to rational mappings.

Finally we remark on a geometric way to view Theorem 1. Consider the polynomial mapping from $\mathbf{C}^{n}$ to $\mathbf{C}^{N}$ defined by $\zeta_{\alpha}(z)=z^{\alpha}$. Here we assume that $N$ equals the dimension of the space of homogeneous polynomials of degree $m$ in $n$ variables. The image of this mapping is an algebraic variety called the Veronese variety $M_{m, n}$. Our theorem has the following reformulation. Suppose that $Q$ is an Hermitian form on $\mathbf{C}^{N}$, and that $Q(\zeta, \bar{\zeta})$ is positive for all $\zeta \in M_{m, n}$ except the origin. By example 2, $Q$ need not be positive everywhere. There is however a Veronese variety $M_{d+m, n}$ of higher degree and an Hermitian form $E^{d} Q$ on it, defined by the process of multiplying the corresponding function by $\|z\|^{2 d}$ and taking the underlying matrix of coefficients, such that the form is positive not only on $M_{d+m, n}$, but on the entire space (except the origin). A moment's thought shows that both conditions are open. Thus the theorem remains true for varieties close to the Veronese. We can think of the Veronese manifold as a testing manifold for positivity. This suggests that there is a generalization of Theorem 1 to more general testing manifolds for positivity.

\section{References}

[D1] J. P. D'Angelo, Several complex variables and the geometry of real hypersurfaces, CRC Press, Boca Raton, 1992.

[D2] J. P. D'Angelo, The geometry of proper holomorphic maps between balls, Contemp. Math. 137 (1992), 191-215.

[F] F. Forstnerič, Extending proper holomorphic mappings of positive codimension, Invent. Math. 95 (1989), 31-62.

[GH] P. Griffith and J. Harris, Principles of algebraic geometry, John Wiley and Sons, New York, 1978.

[HLP] G.H. Hardy, J.E. Littlewood, and G. Polya, Inequalities (1934), University Press, Cambridge.

[H] D. E. Handelman, Positive polynomials, convex integral polytopes, and a random walk problem, Lec. Notes in Math. 1282, Springer-Verlag, Berlin.

[L1] L. Lempert, Imbedding Cauchy-Riemann manifolds into a sphere, International J. Math 1 (1990), 91-108.

[L2] Imbedding pseudoconvex domains into a ball, Amer. J. Math 104 (1982), 901-904.

[L] E. Løw, Embeddings and proper holomorphic maps of strictly pseudoconvex domains into polydiscs and balls, Math. Z. 190 (1985), 401-410. 
[R] B. Reznick, Universal denominators in Hilbert's seventeenth problem, Math. Z. 220 (1995), 75-97.

[Ru] W. Rudin, Functional analysis, McGraw-Hill, New York, 1973.

[W] S.-B. Wono, Proper holomorphic mappings in several complex variables, Ph.D. thesis; Univ. of Illinois (1993).

Department of Mathematics, Purdue University, W. Lafayette, in 47907

E-mail address: catlin@math.purdue.edu

Department of Mathematics, Univerity of Illinois, Urbana, il 61801

E-mail address: jpda@math.uiuc.edu 